\title{
Existence of multiple positive solutions for singular boundary value problems of nonlinear fractional differential equations
}

Wen-Xue Zhou ${ }^{1}{ }^{2 *}$, Jian-Gang Zhang ${ }^{1}$ and Jie-Mei Li ${ }^{1}$

\section{"Correspondence:}

wxzhou2006@126.com

'Department of Mathematics,

Lanzhou Jiaotong University,

Lanzhou, 730070, China

${ }^{2}$ School of Mathematical Sciences,

Fudan University, Shanghai, 200433, China

\begin{abstract}
In this paper, we consider the properties of the Green's function for the nonlinear fractional differential equation boundary value problem $D_{0+}^{q} u(t)=f(t, u(t))$, $t \in J:=[0,1], u(0)=u^{\prime}(1)=0$, where $1<q \leq 2$ is a real number, and $D_{0+}^{q}$ is the standard Riemann-Liouville differentiation. As an application of the Green's function, we give some multiple positive solutions for singular boundary value problems, and we also give the uniqueness of solution for a singular problem by means of the Leray-Schauder nonlinear alternative, a fixed-point theorem on cones, and a mixed monotone method.
\end{abstract}

Keywords: boundary value problem; fractional differential equations; Riemann-Liouville fractional derivative; positive solution; fixed-point theorem

\section{Introduction}

This paper is mainly concerned with the existence and multiplicity of positive solutions of the nonlinear fractional differential equation boundary value problem (BVP for short)

$$
\begin{aligned}
& D_{0^{+}}^{q} u(t)=f(t, u(t)), \quad 0<t<1, \\
& u(0)=u^{\prime}(1)=0,
\end{aligned}
$$

where $1<q \leq 2$ is a real number and $D_{0^{+}}^{q}$ is the standard Riemann-Liouville differentiation, and $f$ is a given function satisfying some assumptions that will be specified later, with $\lim _{u \rightarrow 0} f(\cdot, u)=+\infty$ (i.e., $f$ is singular at $u=0$ ).

In the last few years, fractional differential equations (in short FDEs) have been studied extensively. The motivation for those works stems from both the development of the theory of fractional calculus itself and the applications of such constructions in various sciences such as physics, mechanics, chemistry, engineering, and so on. For an extensive collection of such results, we refer the readers to the monographs by Kilbas et al. [1], Miller and Ross [2], Oldham and Spanier [3], Podlubny [4] and Samko et al. [5].

Some basic theory for the initial value problems of FDE involving Riemann-Liouville differential operator has been discussed by Lakshmikantham [6-8], Babakhani and Daftardar-Gejji [9-11] and Bai [12], and so on. Also, there are some papers which deal with the existence and multiplicity of solutions (or positive solution) for nonlinear FDE

\section{Springer}

○2014 Zhou et al.; licensee Springer. This is an Open Access article distributed under the terms of the Creative Commons Attribution License (http://creativecommons.org/licenses/by/2.0), which permits unrestricted use, distribution, and reproduction in any medium, provided the original work is properly cited. 
of BVPs by using techniques of nonlinear analysis (fixed-point theorems, Leray-Shauder theory, topological degree theory, etc.); see [13-34] and the references therein.

Bai and Lü [15] studied the following two-point boundary value problem of FDEs:

$$
D_{0+}^{q} u(t)+f(t, u(t))=0, \quad u(0)=u(1)=0, \quad 0<t<1,1<q \leq 2,
$$

where $D_{0+}^{q}$ is the standard Riemann-Liouville fractional derivative. They obtained the existence of positive solutions by means of the Guo-Krasnosel'skii fixed-point theorem and the Leggett-Williams fixed-point theorem.

Zhang [23] considered the existence and multiplicity of positive solutions for the nonlinear fractional boundary value problem

$$
{ }^{c} D_{0+}^{q} u(t)=f(t, u(t)), \quad 0<t<1, \quad u(0)+u^{\prime}(0)=0, \quad u(1)+u^{\prime}(1)=0,
$$

where $1<q \leq 2$ is a real number, $f:[0,1] \times[0,+\infty) \rightarrow[0,+\infty)$ and ${ }^{c} D_{0+}^{q}$ is the standard Caputo's fractional derivative. The author obtained the existence and multiplicity results of positive solutions by means of the Guo-Krasnosel'skii fixed-point theorem and the Leggett-Williams fixed-point theorem.

Qiu and Bai [33] considered the existence of positive solutions for the nonlinear fractional boundary value problem

$$
{ }^{c} D_{0+}^{q} u(t)+f(t, u(t))=0, \quad 0<t<1, \quad u(0)=u^{\prime}(1)=u^{\prime \prime}(0)=0,
$$

where $2<q \leq 3$ is a real number, $f:(0,1] \times[0,+\infty) \rightarrow[0,+\infty)$ with $\lim _{t \rightarrow 0^{+}} f(t, \cdot)=\infty$ (i.e., $f$ is singular at $t=0$ ), and ${ }^{c} D_{0+}^{q}$ is the standard Caputo's fractional derivative. The authors proved the existence of one positive solution by using the Guo-Krasnosel'skii fixed-point theorem and the nonlinear alternative of Leray-Schauder type in a cone and assuming certain hypotheses on the function $f$.

Mena et al. [34] proved the existence and uniqueness of a positive and nondecreasing solution for the problem (1.4) by using a fixed-point theorem in partially ordered sets.

From the above works, we can see a fact, although the fractional boundary value problems have been investigated by some authors, singular boundary value problems are seldom considered, in particular, $f$ is singular at $u=0$. Motivated by all the works above, in this paper we discuss the boundary value problem (1.1)-(1.2). Using the Leray-Schauder nonlinear alternative theorem and the Guo-Krasnosel'skii fixed-point theorem, we give some new existence criteria for the singular boundary value problem (1.1)-(1.2). Finally, we obtain new uniqueness criteria for the singular boundary value problem (1.1)-(1.2) by a mixed monotone method.

The plan of this paper is as follows. In Section 2, we shall give some definitions and lemmas to prove our main results. In Section 3, we establish the existence of multiple positive solutions for the singular boundary value problem (1.1)-(1.2) by the Leray-Schauder nonlinear alternative theorem and the Guo-Krasnosel'skii fixed-point theorem. In Section 4, by using a mixed monotone method, we obtain some new uniqueness criteria for the singular boundary value problem (1.1)-(1.2). 


\section{Preliminaries and lemmas}

For the convenience of the reader, we present here the necessary definitions from fractional calculus theory. These definitions can be found in the recent literature such as $[1$, 4] and [15].

Definition 2.1 $[1,4]$ The fractional-order integral of the function $h \in L^{1}\left([a, b], \mathbb{R}_{+}\right)$of order $\alpha \in \mathbb{R}_{+}$is defined by

$$
I_{a}^{\alpha} h(t)=\int_{a}^{t} \frac{(t-s)^{\alpha-1}}{\Gamma(\alpha)} h(s) d s,
$$

where $\Gamma$ is the gamma function. When $a=0$, we write $I^{\alpha} h(t)=\left[h * \varphi_{\alpha}\right](t)$, where $\varphi_{\alpha}(t)=$ $\frac{t^{\alpha-1}}{\Gamma(\alpha)}$ for $t>0$, and $\varphi_{\alpha}(t)=0$ for $t \leq 0$, and $\varphi_{\alpha} \rightarrow \delta(t)$ as $\alpha \rightarrow 0$, where $\delta$ is the delta function.

Definition $2.2[1,4]$ For a function $h$ given on the interval $[a, b]$, the $\alpha$ th RiemannLiouville fractional-order derivative of $h$ is defined by

$$
\left(D_{a+}^{\alpha} h\right)(t)=\frac{1}{\Gamma(n-\alpha)}\left(\frac{d}{d t}\right)^{n} \int_{a}^{t}(t-s)^{n-\alpha-1} h(s) d s,
$$

where $n=[\alpha]+1$ and $[\alpha]$ denotes the integer part of $\alpha$.

From the definition of the Riemann-Liouville derivative, we can obtain the statement.

Lemma 2.1 [15] Let $\alpha>0$. If we assume $u \in C(0,1) \cap L(0,1)$, then differential equation

$$
D_{0^{+}}^{\alpha} u(t)=0,
$$

has

$$
u(t)=C_{1} t^{\alpha-1}+C_{2} t^{\alpha-2}+\cdots+C_{N} t^{\alpha-N}, \quad C_{i} \in \mathbb{R}, i=1,2, \ldots, N,
$$

as unique solutions, where $N$ is the smallest integer greater than or equal to $\alpha$.

Lemma 2.2 [15] Assume that $h \in C(0,1) \cap L(0,1)$ with a derivative of order $q>0$ that belongs to $C(0,1) \cap L(0,1)$. Then

$$
I_{0^{+}}^{q} D_{0^{+}}^{q} h(t)=h(t)+C_{1} t^{\alpha-1}+C_{2} t^{\alpha-2}+\cdots+C_{N} t^{\alpha-N}
$$

for some $C_{i} \in \mathbb{R}, i=1,2, \ldots, N$, where $N$ is the smallest integer greater than or equal to $q$.

In the following, we present the Green's function of the FDE boundary value problem.

Lemma 2.3 Let $h(t) \in C[0,1]$ and $1<q \leq 2$, then the unique solution of

$$
\begin{aligned}
& D_{0^{+}}^{q} u(t)+h(t)=0, \quad 0<t<1, \\
& u(0)=u^{\prime}(1)=0
\end{aligned}
$$


is given by

$$
u(t)=\int_{0}^{1} G(t, s) h(s) d s
$$

where $G(t, s)$ is the Green's function given by

$$
G(t, s)= \begin{cases}\frac{(1-s)^{q-2} t^{q-1}-(t-s)^{q-1}}{\Gamma(q)}, & \text { if } 0 \leq s \leq t \leq 1, \\ \frac{(1-s)^{q-2} t^{q-1}}{\Gamma(q)}, & \text { if } 0 \leq t \leq s \leq 1 .\end{cases}
$$

Proof By Lemma 2.2, we can reduce the equation of problem (2.1) to an equivalent integral equation:

$$
u(t)=-I_{0^{+}}^{q} h(t)+c_{1} t^{q-1}+c_{2} t^{q-2}=-\frac{1}{\Gamma(q)} \int_{0}^{t}(t-s)^{q-1} h(s) d s+c_{1} t^{q-1}+c_{2} t^{q-2}
$$

for some constants $c_{1}, c_{2} \in \mathbb{R}$.

So

$$
u^{\prime}(t)=(q-1) c_{1} t^{q-2}-\frac{q-1}{\Gamma(q)} \int_{0}^{t}(t-s)^{q-2} h(s) d s .
$$

Applying the boundary condition (2.2), we have

$$
c_{1}=\frac{1}{\Gamma(q)} \int_{0}^{1}(1-s)^{q-2} h(s) d s, \quad c_{2}=0 .
$$

Therefore, the unique solution of problem (2.1)-(2.2) is

$$
\begin{aligned}
u(t) & =-\frac{1}{\Gamma(q)} \int_{0}^{t}(t-s)^{q-1} h(s) d s+c_{1} t^{q-1}+c_{2} t^{q-2} \\
& =-\frac{1}{\Gamma(q)} \int_{0}^{t}(t-s)^{q-1} h(s) d s+\frac{1}{\Gamma(q)} \int_{0}^{1}(1-s)^{q-2} t^{q-1} h(s) d s \\
& =\int_{0}^{t}\left[\frac{(1-s)^{q-2} t^{q-1}-(t-s)^{q-1}}{\Gamma(q)}\right] h(s) d s+\int_{t}^{1} \frac{(1-s)^{q-2} t^{q-1}}{\Gamma(q)} h(s) d s \\
& =\int_{0}^{1} G(t, s) h(s) d s,
\end{aligned}
$$

which completes the proof.

The following properties of the Green's function form the basis of our main work in this paper.

Lemma 2.4 Let $k(t)=\frac{t^{q-1}}{\Gamma(q)}, g(s)=\frac{s(1-s)^{q-2}}{\Gamma(q)}$. The function $G(t, s)$ defined by (2.4) satisfies the following conditions:

(i) $\Gamma(q) k(t) g(s) \leq G(t, s) \leq k(t)(1-s)^{q-2}$ for $t, s \in(0,1)$;

(ii) $t^{q-1} g(s) \leq G(t, s) \leq t^{q-2} g(s)$ for $t, s \in(0,1)$;

(iii) $G(t, s)>$ for $t, s \in(0,1)$. 
Proof (i) In the following, we consider $\Gamma(q) G(t, s)$.

When $s \leq t$, we have

$$
\begin{aligned}
\Gamma(q) G(t, s) & =(1-s)^{q-2} t^{q-1}-(t-s)^{q-1} \\
& =t(t-t s)^{q-2}-(t-s)^{q-1} \\
& \geq t(t-t s)^{q-2}-(t-t s)^{q-1} \\
& =t s(t-t s)^{q-2} \\
& =t^{q-1} s(1-s)^{q-2} .
\end{aligned}
$$

On the other hand, we have

$$
\begin{aligned}
\Gamma(q) G(t, s) & =(1-s)^{q-2} t^{q-1}-(t-s)^{q-1} \\
& =t(t-t s)^{q-2}-(t-s)^{q-2}(t-s) \\
& \leq t(t-t s)^{q-2}-(t-t s)^{q-2}(t-s) \\
& =s(t-t s)^{q-2} \\
& =\frac{s}{t} t^{q-1}(1-s)^{q-2} \\
& \leq t^{q-1}(1-s)^{q-2} .
\end{aligned}
$$

When $s \geq t$, we get

$$
\Gamma(q) G(t, s)=(1-s)^{q-2} t^{q-1} \geq s(1-s)^{q-2} t^{q-1} .
$$

On the other hand, we have

$$
\Gamma(q) G(t, s)=(1-s)^{q-2} t^{q-1} .
$$

From (2.6)-(2.9), we have (i).

(ii) When $s \leq t$, we get

$$
\Gamma(q) G(t, s) \geq t^{q-1} s(1-s)^{q-2} .
$$

Thus,

$$
\Gamma(q) G(t, s) t^{2-q}=\left[(1-s)^{q-2} t^{q-1}-(t-s)^{q-1}\right] t^{2-q} \geq t^{q-1} s(1-s)^{q-2} t^{2-q}=t s(1-s)^{q-2} .
$$

On the other hand, we have

$$
\Gamma(q) G(t, s) \leq t^{q-2} s(1-s)^{q-2} .
$$

So

$\Gamma(q) G(t, s) t^{2-q} \leq\left[t^{q-2} s(1-s)^{q-2}\right] t^{2-q}=s(1-s)^{q-2}$. 
When $s \geq t$, we get

$$
\Gamma(q) G(t, s) \geq t^{q-1} s(1-s)^{q-2} .
$$

Thus,

$$
\Gamma(q) G(t, s) t^{2-q} \geq t s(1-s)^{q-2} .
$$

On the other hand, we have

$$
\Gamma(q) G(t, s) t^{q-2}=(1-s)^{q-2} t^{q-1} t^{2-q}=t(1-s)^{q-2} \leq s(1-s)^{q-2} .
$$

Therefore we have (ii). Clearly $G(t, s)>0$ holds trivially. The proof is finished.

Lemma 2.5 The function $G^{*}(t, s):=t^{2-q} G(t, s)$ has the following properties:

$$
\operatorname{tg}(s) \leq G^{*}(t, s) \leq g(s)
$$

where

$$
G^{*}(t, s)= \begin{cases}\frac{\left[(1-s)^{q-2} t^{q-1}-(t-s)^{q-1}\right] t^{2-q}}{\Gamma(q)}, & \text { if } 0 \leq s \leq t \leq 1, \\ \frac{t(1-s)^{q-2}}{\Gamma(q)}, & \text { if } 0 \leq t \leq s \leq 1 .\end{cases}
$$

Let $y(t)=t^{2-q} u(t)$, by $u(t)=\int_{0}^{1} G(t, s) h(s) d s$, we get

$$
y(t)=\int_{0}^{1} t^{2-q} G(t, s) h(s) d s=\int_{0}^{1} G^{*}(t, s) h(s) d s .
$$

The following three theorems are fundamental in the proofs of our main results.

Lemma 2.6 [35] Let $X$ be a Banach space, and let $P \subset X$ be a cone in $X$. Assume $\Omega_{1}, \Omega_{2}$ are open subsets of $X$ with $\theta \in \Omega_{1} \subset \bar{\Omega}_{1} \subset \Omega_{2}$, and let $\mathcal{A}: P \rightarrow P$ be a completely continuous operator such that either

(i) $\|\mathcal{A} u\| \leq\|u\|, u \in P \cap \partial \Omega_{1},\|\mathcal{A} u\| \geq\|u\|, u \in P \cap \partial \Omega_{2}$, or

(ii) $\|\mathcal{A} u\| \geq\|u\|, u \in P \cap \partial \Omega_{1},\|\mathcal{A} u\| \leq\|u\|, u \in P \cap \partial \Omega_{2}$.

Then $\mathcal{A}$ has a fixed point in $P \cap\left(\bar{\Omega}_{2} \backslash \Omega_{1}\right)$.

Let $P$ be a normal cone of a Banach space $E$, and $e \in P$ with $\|e\| \leq 1, e \neq \theta$. Define

$$
Q_{e}=\{x \in P \mid x \neq \theta \text {, there exist constants } m, M>0 \text { such that } m e \leq x \leq M e\} .
$$

Definition 2.3 [36] Assume $A: Q_{e} \times Q_{e} \rightarrow Q_{e} . A$ is said to be mixed monotone if $A(x, y)$ is nondecreasing in $x$ and nonincreasing in $y$, i.e., if $x_{1} \leq x_{2}\left(x_{1}, x_{2} \in Q_{e}\right)$ implies $A\left(x_{1}, y\right) \leq$ $A\left(x_{2}, y\right)$ for any $y \in Q_{e}$, and $y_{1} \leq y_{2}\left(y_{1}, y_{2} \in Q_{e}\right)$ implies $A\left(x, y_{1}\right) \geq A\left(x, y_{2}\right)$ for any $x \in Q_{e}$. $x^{*} \in Q_{e}$ is said to be a fixed point of $A$ if $A\left(x^{*}, x^{*}\right)=x^{*}$. 
Lemma 2.7 [36] Suppose that $A: Q_{e} \times Q_{e} \rightarrow Q_{e}$ is a mixed monotone operator and $\exists a$ constant $\beta(0 \leq \beta<1)$ such that

$$
A\left(t x, \frac{1}{t} y\right) \geq t^{\beta} A(x, y), \quad \forall x, y \in Q_{e}, 0<t<1 .
$$

Then $A$ has a unique fixed point $x^{*} \in Q_{e}$.

Lemma 2.8 [37] Assume $\Omega$ is a relative subset of a convex set $K$ in a normed space X. Let $A: \bar{\Omega} \rightarrow K$ be a compact map with $0 \in \Omega$. Then either

(A1) $A$ has a fixed point in $\bar{\Omega}$, or

(A2) there is $a x \in \partial \Omega$ and $a \lambda<1$ such that $x=\lambda A(x)$.

\section{Positive solutions of a singular problem}

In this section, we establish some new existence results for the singular fractional differential equation (1.1)-(1.2). We always assume that $f:[0,1] \times(0, \infty) \rightarrow[0, \infty)$ is continuous in this section. Given $a \in L^{1}(0,1)$, we write a $a \succ 0$ if $a \geq 0$ for $t \in[0,1]$ and it is positive in a set of positive measure.

Theorem 3.1 Suppose that the following hypotheses hold:

(H1) for each constant $L>0$, there exists a continuous function $\phi_{L} \succ 0$ such that $f(t, x) \geq \phi_{L}(t)$; for all $t \in[0,1]$ and $x \in(0, L]$, one has $0<\int_{0}^{1} g(s) \phi_{L}(s) d s<\infty$;

$(\mathrm{H} 2)$ there exist continuous, nonnegative functions $b(x)$ and $d(x)$ such that

$$
0 \leq f(t, x)=b(x)+d(x) \quad \text { for all }(t, x) \in[0,1] \times(0, \infty),
$$

and $b(x)>0$ is nonincreasing and $\frac{d(x)}{b(x)}$ is nondecreasing in $x \in(0, \infty)$;

(H3) there exists a constant $K_{0}>0$ such that $b(l m) \leq K_{0} b(l) b(m)$ for all $l, m \geq 0$;

(H4) $\int_{0}^{1} b\left(s^{q-1}\right) d s<\infty$;

(H5) there exists a constant $r>0$ such that

$$
(b(r)+d(r)) K_{0} \int_{0}^{1} g(s) t^{q-2} b(s) d s<r .
$$

Then problem (1.1)-(1.2) has at least one positive solution $x$ with $0<\|x\|<r$.

Proof Since (H5) holds, we can choose $n_{0} \in\{1,2, \ldots\}$ such that

$$
(b(r)+d(r)) K_{0} \int_{0}^{1} g(s) t^{q-2} b(s) d s+\frac{1}{n_{0}}<r .
$$

Let $N_{0}=\left\{n_{0}, n_{0}+1, \ldots\right\}$. Fix $n \in N_{0}$ and consider the family of integral equations

$$
u(t):=\lambda \int_{0}^{1} G(t, s) f_{n}(s, u(s)) d s+\frac{1}{n},
$$

where $\lambda \in[0,1]$ and

$$
f_{n}(t, u)= \begin{cases}f(t, u), & \text { if } u \geq \frac{1}{n} \\ f\left(t, \frac{1}{n}\right), & \text { if } u \leq \frac{1}{n}\end{cases}
$$


We claim that any solution $u$ of (3.1) for any $\lambda \in[0,1]$ must satisfy $\|u\| \neq r$. Otherwise, assume that $u$ is a solution of (3.1) for some $\lambda \in[0,1]$ such that $\|u\|=r$. Then $u(t) \geq \frac{1}{n}$ for $t \in[0,1]$. Note that

$$
\|u\| \leq \frac{1}{n}+\lambda \int_{0}^{1} g(s) t^{q-2} f_{n}(s, u(s)) d s
$$

Hence, for all $t \in[0,1]$, we have

$$
\begin{aligned}
u(t) & \geq \frac{1}{n}+\lambda \int_{0}^{1} g(s) t^{q-1} f_{n}(s, u(s)) d s \\
& \geq \frac{1}{n}+t^{q-1}\left(\|u\|-\frac{1}{n}\right) \\
& \geq t^{q-1}\|u\|=t^{q-1} r .
\end{aligned}
$$

Thus we have from condition (H2), for all $t \in[0,1]$,

$$
\begin{aligned}
u(t) & =\lambda \int_{0}^{1} G(t, s) f_{n}(s, u(s)) d s+\frac{1}{n} \\
& =\lambda \int_{0}^{1} G(t, s) f(s, u(s)) d s+\frac{1}{n} \\
& \leq \int_{0}^{1} g(s) t^{q-2} f(s, u(s)) d s+\frac{1}{n} \\
& \leq \int_{0}^{1} g(s) t^{q-2} b(u(s))\left(1+\frac{d(u(s))}{b(u(s))}\right) d s+\frac{1}{n} \\
& \leq\left(1+\frac{d(r)}{b(r)}\right) \int_{0}^{1} g(s) t^{q-2} K_{0} b(r) b(s) d s+\frac{1}{n} \\
& \leq(b(r)+d(r)) K_{0} \int_{0}^{1} g(s) t^{q-2} b(s) d s+\frac{1}{n_{0}} .
\end{aligned}
$$

Therefore,

$$
r=\|u(t)\| \leq(b(r)+d(r)) K_{0} \int_{0}^{1} g(s) t^{q-2} b(s) d s+\frac{1}{n_{0}} .
$$

This is a contradiction and the claim is proved.

Now the Leray-Schauder nonlinear alternative guarantees that the integral equation

$$
u(t)=\int_{0}^{1} G(t, s) f_{n}(s, u(s)) d s+\frac{1}{n}
$$

has a solution, denoted by $u_{n}$, in $\bar{B}_{r}=\{x \in C(J):\|u\| \leq r\}$.

Next we claim that $u_{n}(t)$ has a uniform sharper lower bound, i.e., there exists a function $\rho \in C([0,1])$ that is unrelated to $n$ such that $\rho(t)>0$ for a.e. $t \in[0,1]$ and for any $n \in N_{0}$,

$$
u_{n}(t) \geq \rho(t), \quad t \in[0,1]
$$


By $(\mathrm{H} 1)$, there exists a continuous function $\phi_{r} \succ 0$ such that $f(t, x) \geq \phi_{r}(t)$ for all $t \in[0,1]$ and $\|x\| \leq r$. In view of $u_{n}(t) \leq r$, so we have

$$
\begin{aligned}
u_{n}(t) & =\int_{0}^{1} G(t, s) f_{n}\left(s, u_{n}(s)\right) d s+\frac{1}{n} \\
& =\int_{0}^{1} G(t, s) f\left(s, u_{n}(s)\right) d s+\frac{1}{n} \\
& \geq \int_{0}^{1} G(t, s) \phi_{r}(s) d s \\
& \geq t^{q-1} \int_{0}^{1} g(s) \phi_{r}(s) d s .
\end{aligned}
$$

We choose $\rho(t)=t^{q-1} \int_{0}^{1} g(s) \phi_{r}(s) d s$. Then (3.5) holds.

In order to pass from the solutions $u_{n}$ of the truncation equation (3.4) to that of the original equation (1.1)-(1.2), we need the following fact:

$\left\{u_{n}\right\}_{n \in N_{0}}$ is an equicontinuous family on $[0,1]$.

In fact, for any $t_{1}, t_{2} \in[0,1]$, we have

$$
\begin{aligned}
\left|u_{n}\left(t_{1}\right)-u_{n}\left(t_{2}\right)\right| & =\left|\int_{0}^{1}\left[G\left(t_{1}, s\right)-G\left(t_{2}, s\right)\right] f\left(s, u_{n}(s)\right) d s\right| \\
& \leq \int_{0}^{1}\left|G\left(t_{1}, s\right)-G\left(t_{2}, s\right)\right| f\left(s, u_{n}(s)\right) d s \\
& \leq\left(1+\frac{d(r)}{b(r)}\right) \int_{0}^{1}\left|G\left(t_{1}, s\right)-G\left(t_{2}, s\right)\right| b\left(s \int_{0}^{1} g(\tau) \phi_{r}(\tau) d \tau\right) d s \\
& \leq\left(1+\frac{d(r)}{b(r)}\right) \int_{0}^{1}\left|G\left(t_{1}, s\right)-G\left(t_{2}, s\right)\right| K_{0} b(s) b\left(\int_{0}^{1} g(\tau) \phi_{r}(\tau) d \tau\right) d s \\
& \leq\left(1+\frac{d(r)}{b(r)}\right) K_{0} b\left(\int_{0}^{1} g(\tau) \phi_{r}(\tau) d \tau\right) \int_{0}^{1}\left|G\left(t_{1}, s\right)-G\left(t_{2}, s\right)\right| b\left(s^{q-1}\right) d s
\end{aligned}
$$

By continuity of $G(t, \cdot)$ and the mean value theorem for integrals, there exists a $\xi \in(0,1)$ such that

$$
\left|u_{n}\left(t_{1}\right)-u_{n}\left(t_{2}\right)\right| \leq\left(1+\frac{d(r)}{b(r)}\right) K_{0} b\left(\int_{0}^{1} g(\tau) \phi_{r}(\tau) d \tau\right) \int_{0}^{1}\left|G\left(t_{1}, \xi\right)-G\left(t_{2}, \xi\right)\right| b(s) d s
$$

By the continuity of $G(\cdot, s)$ and (H4), then (3.6) holds. By the Arzela-Ascoli theorem, there exist a subsequence $N_{1}$ of $N_{0}$ and $u \in C([0,1])$ such that $\left\{u_{n}\right\}_{n \in N_{1}}$ is uniformly convergent to $u$ and $u$ satisfies $\rho(t) \leq u(t) \leq r$ for any $t \in[0,1]$. In view of $u_{n}(t)=$ $\int_{0}^{1} G(t, s) f_{n}\left(s, u_{n}(s)\right) d s$, by the Lebesgue dominated convergence theorem, we have $u(t)=$ $\int_{0}^{1} G(t, s) f(s, u(s)) d s$. Therefore, (1.1)-(1.2) have one positive solution $u$ with $0<\|u\|<r$. This completes the proof.

Theorem 3.2 Suppose that (H2), (H3), (H4), and (H5) are satisfied. Furthermore assume that: 
(H6) There exists a positive number $R>r$ such that

$$
\frac{1}{\Gamma(q)}\left(1-\frac{1}{q}\right)^{q-1} b(R) \int_{0}^{1} s(1-s)^{q-1}\left\{1+\frac{d(s R)}{b(s R)}\right\} d s \geq R
$$

then problem (1.1)-(1.2) has a solution $\tilde{u}$ with $r<\|\tilde{u}\| \leq R$.

Proof To show the existence of $\widetilde{u}$, we will use Lemma 2.6. Define

$$
K=\{u \in C[0,1]: u(t) \geq t\|u\|, \forall t \in[0,1]\} .
$$

Clearly $K$ is a cone of $C[0,1]$. Let

$$
\Omega_{1}=\{u \in C[0,1]:\|u\|<r\}, \quad \Omega_{2}=\{u \in C[0,1]:\|u\|<R\} .
$$

Next, let $\mathcal{A}: K \cap\left(\bar{\Omega}_{2} \backslash \Omega_{1}\right) \rightarrow C[0,1]$ be defined by

$$
(\mathcal{A} y)(t):=\int_{0}^{1} G^{*}(t, s) f\left(s, s^{q-2} y(s)\right) d s
$$

First we show that $\mathcal{A}$ maps $K \cap\left(\bar{\Omega}_{2} \backslash \Omega_{1}\right)$. If $y \in K \cap\left(\bar{\Omega}_{2} \backslash \Omega_{1}\right)$, then for $t \in[0,1]$ we have

$$
(\mathcal{A} y)(t) \leq \frac{1}{\Gamma(q)} \int_{0}^{1} s(1-s)^{q-2} f\left(s, s^{q-2} y(s)\right) d s
$$

and

$$
(\mathcal{A} y)(t) \geq \frac{t}{\Gamma(q)} \int_{0}^{1} s(1-s)^{q-2} f\left(s, s^{q-2} y(s)\right) d s
$$

this implies that $(\mathcal{A} y)(t) \geq t\|\mathcal{A} y\|$, i.e. $\mathcal{A} y \in K$.

Next, we show that $\mathcal{A}$ is equicontinuous. The proof will be given in several steps.

Step 1: We will show that $\mathcal{A}$ is continuous.

In fact, let $x_{n}, x \in K \cap\left(\bar{\Omega}_{2} \backslash \Omega_{1}\right), n=1,2,3, \ldots$ with $\lim _{n \rightarrow \infty}\left\|x_{n}-x\right\|=0$. It is obvious that $r<\left\|x_{n}\right\| \leq R, r<\|x\| \leq R, x_{n}(t) \geq t r, x(t) \geq \operatorname{tr}$. We have

$$
\begin{aligned}
& x_{n}(t) \in[t r, R], \quad n \in\{1,2, \ldots\}, t \in[0,1], \\
& x(t) \in[\operatorname{tr}, R], \quad t \in[0,1] .
\end{aligned}
$$

Notice also that

$$
\gamma_{n}(s)=\left\|f\left(s, x_{n}(s)\right)-f(s, x(s))\right\| \rightarrow 0, \quad \text { as } n \rightarrow \infty, \text { for } \forall s \in[0,1]
$$

and

$$
\gamma_{n}(s) \leq 2 b(t r)\left\{1+\frac{d(R)}{b(R)}\right\} \leq 2 K_{0} b(t) b(r)\left\{1+\frac{d(R)}{b(R)}\right\} .
$$


Now these together with the Lebesgue dominated convergence theorem guarantee that

$$
\left\|\left(\mathcal{A} x_{n}\right)(t)-(\mathcal{A} x)(t)\right\| \leq(q-1) \int_{0}^{1} q(s) \gamma_{n}(s) d s \rightarrow 0, \quad \text { as } n \rightarrow \infty .
$$

Hence $\mathcal{A}: K \cap\left(\bar{\Omega}_{2} \backslash \Omega_{1}\right) \rightarrow K$ is continuous.

Step 2: We will prove that the operator $\mathcal{A}: K \cap\left(\bar{\Omega}_{2} \backslash \Omega_{1}\right) \rightarrow K$ is compact.

Indeed, for $x \in K \cap\left(\bar{\Omega}_{2} \backslash \Omega_{1}\right)$,

$$
\begin{aligned}
\|\mathcal{A} x\| & \leq(q-1)\left\{1+\frac{d(R)}{b(R)}\right\} \int_{0}^{1} q(s) b(s r) d s \\
& \leq(q-1)\left\{1+\frac{d(R)}{b(R)}\right\} K_{0} b(r) \int_{0}^{1} q(s) b(s) d s,
\end{aligned}
$$

and for $t, t^{\prime} \in[0,1]$, we have

$$
\begin{aligned}
\left\|\mathcal{A} x(t)-\mathcal{A} x\left(t^{\prime}\right)\right\| & \leq\left\{1+\frac{d(R)}{b(R)}\right\} \int_{0}^{1}\left|G(t, s)-G\left(t^{\prime}, s\right)\right| b(s r) d s \\
& \leq\left\{1+\frac{d(R)}{b(R)}\right\} K_{0} b(r) \int_{0}^{1}\left|G(t, s)-G\left(t^{\prime}, s\right)\right| b(s) d s .
\end{aligned}
$$

By continuity of $G(t, \cdot)$ and the mean value theorem for integrals, there exists a $\eta \in(0,1)$ such that

$$
\left\|\mathcal{A} x(t)-\mathcal{A} x\left(t^{\prime}\right)\right\| \leq\left\{1+\frac{d(R)}{b(R)}\right\} K_{0} b(r)\left|G(t, \eta)-G\left(t^{\prime}, \eta\right)\right| \int_{0}^{1} b(s) d s .
$$

By continuity of $G(\cdot, s)$, using condition (H4), and the Arzela-Ascoli theorem guarantees that $\mathcal{A}: K \cap\left(\bar{\Omega}_{2} \backslash \Omega_{1}\right) \rightarrow K$ is compact.

Now we prove that

$$
\|\mathcal{A} x\| \leq\|x\|, \quad \forall x \in K \cap \partial \Omega_{1} .
$$

In fact, for any $x \in K \cap \partial \Omega_{1}$, we have for $t \in[0,1]$,

$$
\begin{aligned}
\mathcal{A} x(t) & =\int_{0}^{1} G(t, s) f(s, x(s)) d s \\
& \leq \int_{0}^{1} G(t, s) b(x(s))\left\{1+\frac{d(x(s))}{b(x(s))}\right\} d s \\
& \leq\left\{1+\frac{d(r)}{b(r)}\right\} \int_{0}^{1} g(s) t^{q-2} b(s r) d s \\
& \leq\left\{1+\frac{d(r)}{b(r)}\right\} K_{0} b(r) \int_{0}^{1} g(s) t^{q-2} b(s) d s \\
& =\{b(r)+d(r)\} K_{0} \int_{0}^{1} g(s) t^{q-2} b(s) d s \\
& <r \\
& =\|x\| .
\end{aligned}
$$


Therefore, $\|\mathcal{A} x\| \leq\|x\|$, i.e., (3.9) holds. On the other hand, we prove that

$$
\|\mathcal{A} x\| \geq\|x\|, \quad \forall x \in K \cap \partial \Omega_{2} .
$$

In fact, for any $x \in K \cap \partial \Omega_{2}$, we have for $t \in[0,1]$,

$$
\begin{aligned}
\mathcal{A} x\left(1-\frac{1}{q}\right) & =\int_{0}^{1} G\left(1-\frac{1}{q}, s\right) f(s, x(s)) d s \\
& \geq \frac{1}{\Gamma(q)}\left(1-\frac{1}{q}\right)^{q-1} \int_{0}^{1} s(1-s)^{q-1} b(x(s))\left\{1+\frac{d(x(s))}{b(x(s))}\right\} d s \\
& \geq \frac{1}{\Gamma(q)}\left(1-\frac{1}{q}\right)^{q-1} b(R) \int_{0}^{1} s(1-s)^{q-1}\left\{1+\frac{d(s R)}{b(s R)}\right\} d s \\
& \geq R \\
& =\|x\| .
\end{aligned}
$$

This implies (3.10) holds.

It follows from Lemma 2.6, (3.9), and (3.10) that $\mathcal{A}$ has a fixed point $K \cap\left(\bar{\Omega}_{2} \backslash \Omega_{1}\right)$. Clearly, this fixed point is a positive solution of (1.1)-(1.2) satisfying $r<\|\widetilde{u}\| \leq R$. This completes the proof.

Theorem 3.3 Suppose that (H1)-(H6) are satisfied. Then problem (1.1)-(1.2) has two solutions $u$ and $\widetilde{u}$ with $0<\|u\|<r<\|\widetilde{u}\| \leq R$.

\section{Uniqueness of solution for a singular problem}

Throughout this section we assume that

(H7) $f(t, x)=q_{1}(t)\left[g_{1}(x)+h_{1}(x)\right], t \in(0,1)$, where

$$
\begin{aligned}
& g_{1}:[0,+\infty) \rightarrow[0,+\infty) \text { is continuous and nondecreasing; } \\
& h_{1}:(0,+\infty) \rightarrow(0,+\infty) \text { is continuous and nonincreasing. }
\end{aligned}
$$

By property (i) of the Green's function in Lemma 2.4, we assume there exist $a, m, n \in C[0,1]$ with $a(t), m(t), n(t)>0$ for $t \in(0,1)$ such that

$$
a(t) m(s) \leq G(t, s) \Gamma(q) \leq a(t) n(s), \quad t, s \in[0,1],
$$

where $a(t)=t^{q-1}, m(s)=s(1-s)^{q-2}, n(s)=(1-s)^{q-2}$. Clearly $\|a\|=\sup _{t \in J} a(t)<1$.

Suppose that $x$ is a solution of (1.1)-(1.2), then

$$
x(t):=\int_{0}^{1} G(t, s) f(s, x(s)) d s, \quad t \in[0,1] .
$$

By (4.1), we have

$$
a(t) \int_{0}^{1} \frac{1}{\Gamma(q)} m(s) f(s, x(s)) d s \leq x(t) \leq a(t) \int_{0}^{1} \frac{1}{\Gamma(q)} n(s) f(s, x(s)) d s
$$


So if $x(t)$ is a solution of problem (1.1)-(1.2), then $x \in Q_{e}$ which was defined in (2.10), where $e(t)=t^{q-1}=a(t)$.

Let $P=\{x \in C[0,1]: x(t) \geq 0, \forall t \in[0,1]\}$. Clearly $P$ is a normal cone of the Banach space $C[0,1]$.

Theorem 4.1 Suppose that (H7) is satisfied, and there exists $\beta \in(0,1)$ such that

$$
g_{1}(t x) \geq t^{\beta} g_{1}(x)
$$

and

$$
h_{1}\left(t^{-1} x\right) \geq t^{\beta} h_{1}(x)
$$

for any $t \in(0,1)$ and $x>0$, and $q_{1} \in C((0,1),(0, \infty))$ satisfies

$$
\int_{0}^{1} \frac{1}{\Gamma(q)} n(s) a^{-\beta}(s) q_{1}(s) d s<+\infty ;
$$

then problem (1.1)-(1.2) has a unique positive solution $x^{*}$.

Proof Since (4.3) holds, let $t^{-1} x=y$; one has

$$
h_{1}(y) \geq t^{\beta} h_{1}(t y) .
$$

Then

$$
h_{1}(t y) \leq t^{-\beta} h_{1}(y), \quad \forall t \in(0,1), y>0 .
$$

Let $y=1$. The above inequality is

$$
h_{1}(t) \leq t^{-\beta} h_{1}(1), \quad \forall t \in(0,1) .
$$

From (4.3), (4.5), and (4.6), one has

$$
\begin{aligned}
& h_{1}\left(t^{-1} x\right) \geq t^{\beta} h_{1}(x), \quad h_{1}\left(\frac{1}{t}\right) \geq t^{\beta} h_{1}(1), \quad h_{1}(t x) \leq t^{-\beta} h_{1}(x), \\
& h_{1}(t) \leq t^{-\beta} h_{1}(1), \quad t \in(0,1), x>0 .
\end{aligned}
$$

Similarly, from (4.2), one has

$$
g_{1}(t x) \geq t^{\beta} g_{1}(x), \quad g_{1}(t) \geq t^{\beta} g_{1}(1), \quad t \in(0,1), x>0 .
$$

Let $t=\frac{1}{x}, x>1$, so one has

$$
g_{1}(x) \leq x^{\beta} g_{1}(1), \quad t \geq 1 .
$$

Let $e(t)=a(t)$, and we define

$$
Q_{e}=\left\{x \in P \mid \frac{1}{M} a(t) \leq x(t) \leq M a(t), t \in[0,1]\right\},
$$


where $M>1$ is chosen such that

$$
\begin{gathered}
M> \\
\max \left\{\left\{\int_{0}^{1} \lambda \frac{1}{\Gamma(q)} n(s) q_{1}(s) a^{-\beta}(s)\left[g_{1}(1)+h_{1}(1)\right] d s\right\}^{\frac{1}{1-\beta}},\right. \\
\left.\left\{\int_{0}^{1} \lambda \frac{1}{\Gamma(q)} m(s) q_{1}(s) a^{\beta}(s)\left[g_{1}(1)+h_{1}(1)\right] d s\right\}^{-\frac{1}{1-\beta}}\right\} .
\end{gathered}
$$

For any $x, y \in Q_{e}$ we define

$$
A_{\lambda}(x, y)(t)=\lambda \int_{0}^{1} G(t, s) q_{1}(s)\left[g_{1}(x(s))+h_{1}(y(s))\right] d s, \quad \forall t \in[0,1] .
$$

First we show that $A_{\lambda}: Q_{e} \times Q_{e} \rightarrow Q_{e}$. Let $x, y \in Q_{e}$ and from (4.8) we have

$$
g_{1}(x(t)) \leq g_{1}(M a(t)) \leq g_{1}(M) \leq M^{\beta} g_{1}(1), \quad t \in(0,1),
$$

and from (4.7) we have

$$
h_{1}(y(t)) \leq h_{1}\left(\frac{1}{M} a(t)\right) \leq a^{-\beta}(t) h_{1}\left(\frac{1}{M}\right) \leq M^{\beta} a^{-\beta}(t) h_{1}(1), \quad t \in(0,1) .
$$

So we have

$$
\begin{aligned}
A_{\lambda}(x, y)(t) & \leq \lambda \int_{0}^{1} \frac{1}{\Gamma(q)} a(t) n(s) q_{1}(s)\left[g_{1}(x(s))+h_{1}(y(s))\right] d s \\
& \leq \lambda a(t) M^{\beta} \int_{0}^{1} \frac{1}{\Gamma(q)} n(s) q_{1}(s)\left[g_{1}(1)+a^{-\beta}(s) h_{1}(1)\right] d s \\
& \leq \lambda a(t) M^{\beta} \int_{0}^{1} \frac{1}{\Gamma(q)} n(s) q_{1}(s)\left[g_{1}(1)+a^{-\beta} h_{1}(1)\right] d s \\
& \leq \lambda a(t) M^{\beta} \int_{0}^{1} \frac{1}{\Gamma(q)} n(s) q_{1}(s) a^{-\beta}(s)\left[g_{1}(1)+h_{1}(1)\right] d s \\
& \leq M a(t), \quad \forall t \in[0,1] .
\end{aligned}
$$

On the other hand, for any $x, y \in Q_{e}$, from (4.7) and (4.8), we have

$$
g_{1}(x(t)) \geq g_{1}\left(\frac{1}{M} a(t)\right) \geq a^{\beta}(t) g_{1}\left(\frac{1}{M}\right) \geq a^{\beta}(t) \frac{1}{M^{\beta}} g_{1}(1), \quad t \in(0,1)
$$

and

$$
h_{1}(y(t)) \geq h_{1}(M a(t)) \geq h_{1}(M)=h_{1}\left(\frac{1}{\frac{1}{M}}\right) \geq \frac{1}{M^{\beta}} h_{1}(1), \quad t \in(0,1),
$$

so we have

$$
\begin{aligned}
A_{\lambda}(x, y)(t) & \geq \lambda \int_{0}^{1} \frac{1}{\Gamma(q)} a(t) m(s) q_{1}(s)\left[g_{1}(x(s))+h_{1}(y(s))\right] d s \\
& \geq \lambda a(t) M^{-\beta} \int_{0}^{1} \frac{1}{\Gamma(q)} m(s) q_{1}(s)\left[a^{\beta}(s) g_{1}(1)+h_{1}(1)\right] d s
\end{aligned}
$$




$$
\begin{aligned}
& \geq \lambda a(t) M^{-\beta} \int_{0}^{1} \frac{1}{\Gamma(q)} m(s) q_{1}(s) a^{\beta}(s)\left[g_{1}(1)+h_{1}(1)\right] d s \\
& \geq \frac{1}{M} a(t), \quad \forall t \in[0,1] .
\end{aligned}
$$

Thus $A_{\lambda}$ is well defined and $A_{\lambda}\left(Q_{e} \times Q_{e}\right) \subseteq Q_{e}$.

Next, for any $l \in(0,1)$ and $x, y \in Q_{e}$ we have

$$
\begin{aligned}
A_{\lambda}\left(l x, l^{-1} y\right)(t) & =\lambda \int_{0}^{1} G(t, s) q_{1}(s)\left[g_{1}(l x(s))+h_{1}\left(l^{-1} y(s)\right)\right] d s \\
& \geq \lambda \int_{0}^{1} G(t, s) q_{1}(s)\left[l^{\beta} g_{1}(x(s))+l^{\beta} h_{1}(y(s))\right] d s \\
& =l^{\beta} A(x, y)(t), \quad \forall t \in[0,1] .
\end{aligned}
$$

Thus the conditions of Lemma 2.7 hold. Therefore there exists a unique $x^{*} \in Q_{e}$ such that $A_{\lambda}\left(x^{*}, x^{*}\right)=x^{*}$. This completes the proof.

Example 1 Consider the boundary value problem

$$
\begin{aligned}
& D_{0^{+}}^{q} u(t)=u^{-a}(t)+v u^{b}(t), \quad 0<t<1, \\
& u(0)=u^{\prime}(1)=0,
\end{aligned}
$$

where $0<a, b<1, v \geq 0$.

We let

$$
\beta=\max \{a, b\}<1, \quad q_{1}(t)=1, \quad g_{1}(x)=v x^{b}, \quad h_{1}(x)=x^{-a} .
$$

Thus, we have

$$
g_{1}(t x)=t^{b} g_{1}(x) \geq t^{\beta} g_{1}(x), \quad h_{1}\left(t^{-1} x\right)=t^{a} h_{1}(x) \geq t^{\beta} h_{1}(x) .
$$

For any $t \in(0,1)$ and $x>0$, and

$$
\int_{0}^{1} \frac{1}{\Gamma(q)} n(s) a^{-\beta}(s) q_{1}(s) d s<+\infty .
$$

Since $\beta<1$, and $1<q \leq 2$, thus all conditions in Theorem 4.1 are satisfied. Applying Theorem 4.1, we can find that (4.13)-(4.14) has a unique positive solution $x^{*}(t)$.

\section{Competing interests}

The authors declare that they have no competing interests.

\section{Authors' contributions}

All authors contributed equally to the manuscript. All authors read and approved the final manuscript.

\section{Acknowledgements}

This research was supported by the National Natural Science Foundation of China (11161027, 11262009); by the Scientific Research Projects in Colleges and Universities of Gansu Province of China (2013A-043); by the Fundamental Research Funds for the Gansu Universities; by the Fundamental Research Funds for the Gansu Universities (212084); by the Youth Science Foundation of Lanzhou Jiaotong University (2012019); by the National Natural Science Foundation of China (11226132). The authors are thankful the referees for their careful reading of the manuscript and insightful comments. 


\section{References}

1. Kilbas, AA, Srivastava, HM, Trujillo, JJ: Theory and Applications of Fractional Differential Equations. Elsevier, Amsterdam (2006)

2. Miller, KS, Ross, B: An Introduction to the Fractional Calculus and Differential Equations. Wiley, New York (1993)

3. Oldham, KB, Spanier, J: The Fractional Calculus. Academic Press, New York (1974)

4. Podlubny, I: Fractional Differential Equations. Academic Press, San Diego (1999)

5. Samko, SG, Kilbas, AA, Marichev, Ol: Fractional Integrals and Derivatives, Theory and Applications. Gordon \& Breach Yverdon (1993)

6. Lakshmikantham, V, Vatsala, AS: Basic theory of fractional differential equations. Nonlinear Anal. 69(8), 2677-2682 (2008)

7. Lakshmikantham, $\vee$, Vatsala, AS: General uniqueness and monotone iterative technique for fractional differential equations. Appl. Math. Lett. 21(8), 828-834 (2008)

8. Lakshmikantham, V: Theory of fractional functional differential equations. Nonlinear Anal. 69(10), 3337-3343 (2008)

9. Babakhani, A, Daftardar-Gejji, V: Existence of positive solutions of nonlinear fractional differential equations. J. Math. Anal. Appl. 278(2), 434-442 (2003)

10. Babakhani, A, Daftardar-Gejji, V: Existence of positive solutions for $N$-term non-autonomous fractional differential equations. Positivity 9, 193-206 (2005)

11. Babakhani, A, Daftardar-Gejji, V: Existence of positive solutions for multi-term non-autonomous fractional differential equations with polynomial coefficients. Electron. J. Differ. Equ. 2006, 129 (2006)

12. Bai, C-Z: Positive solutions for nonlinear fractional differential equations with coefficient that changes sign. Nonlinear Anal. 64(4), 677-685 (2006)

13. Agarwal, RP, Benchohra, M, Hamani, S: Boundary value problems for fractional differential equations. Adv. Stud. Contemp. Math. 16(2), 181-196 (2008)

14. Ahmad, B, Nieto, JJ: Existence results for nonlinear boundary value problems of fractional integrodifferential equations with integral boundary conditions. Bound. Value Probl. 2009, Article ID 708576 (2009)

15. Bai, ZB, Lü, H: Positive solutions for boundary value problem of nonlinear fractional differential equation. J. Math. Anal. Appl. 311(2), 495-505 (2005)

16. Benchohra, M, Graef, JR, Hamani, S: Existence results for boundary value problems with nonlinear fractional differential equations. Appl. Anal. 87(7), 851-863 (2008)

17. Benchohra, M, Hamani, S, Ntouyas, SK: Boundary value problems for differential equations with fractional order. Surv. Math. Appl. 3, 1-12 (2008)

18. Benchohra, M, Henderson, J, Ntouyas, SK, Ouahab, A: Existence results for fractional order functional differential equations with infinite delay. J. Math. Anal. Appl. 338(2), 1340-1350 (2008)

19. Daftardar-Gejji, V: Positive solutions of a system of non-autonomous nonlinear fractional differential equations. J. Math. Anal. Appl. 302(1), 56-64 (2005)

20. Kaufmann, ER, Mboumi, E: Positive solutions of a boundary value problem for a nonlinear fractional differential equation. Electron. J. Qual. Theory Differ. Equ. 2008, 3 (2008)

21. Yu, C, Gao, G-Z: Existence of fractional differential equations. J. Math. Anal. Appl. 310(1), 26-29 (2005)

22. Zhang, S-Q: The existence of a positive solution for a nonlinear fractional differential equation. J. Math. Anal. Appl. 252(2), 804-812 (2000)

23. Zhang, S-Q: Positive solutions for boundary-value problems of nonlinear fractional differential equations. Electron. J. Differ. Equ. 2006, 36 (2006)

24. Baleanu, D, Agarwal, RP, Mohammadi, H, Rezapour, S: Some existence results for a nonlinear fractional differential equation on partially ordered Banach spaces. Bound. Value Probl. 2013, 112 (2013)

25. Baleanu, D, Mohammadi, H, Rezapour, S: Positive solutions of a boundary value problem for nonlinear fractional differential equations. Abstr. Appl. Anal. 2012, Article ID 837437 (2012)

26. Baleanu, D, Mohammadi, H, Rezapour, S: Some existence results on nonlinear fractional differential equations. Philos. Trans. R. Soc. Lond. A 371, 20120144 (2013)

27. Baleanu, D, Mohammadi, H, Rezapour, S: On a nonlinear fractional differential equation on partially ordered metric spaces. Adv. Differ. Equ. 2013, 83 (2013)

28. Zhou, W-X, Chu, Y-D: Existence of solutions for fractional differential equations with multi-point boundary conditions Commun. Nonlinear Sci. Numer. Simul. 17(3), 1142-1148 (2012)

29. Zhou, W-X, Chu, Y-D, Baleanu, D: Uniqueness and existence of positive solutions for a multi-point boundary value problem of singular fractional differential equations. Adv. Differ. Equ. 2013, 114 (2013)

30. Zhou, W-X, Chang, Y-X, Liu, H-Z: Weak solutions for nonlinear fractional differential equations in Banach spaces Discrete Dyn. Nat. Soc. 2012, Article ID 527969 (2012)

31. Zhou, W-X, Liu, H-Z: Existence of weak solutions for nonlinear fractional differential inclusion with non-separated boundary conditions. J. Appl. Math. 2012, Article ID 530624 (2012)

32. Zhou, W-X, Peng, J-G, Chu, Y-D: Multiple positive solutions for nonlinear semipositone fractional differential equations. Discrete Dyn. Nat. Soc. 2012, Article ID 850871 (2012)

33. Qiu, T-T, Bai, Z-B: Existence of positive solutions for singular fractional differential equations. Electron. J. Differ. Equ. 2008, $146(2008)$

34. Mena, JC, Harjani, J, Sadarangani, K: Existence and uniqueness of positive and nondecreasing solutions for a class of singular fractional boundary value problems. Bound. Value Probl. 2009, Article ID 421310 (2009)

35. Guo, D-J: The Order Methods in Nonlinear Analysis. Shandong Technical and Science Press, Jinan (2000) (in Chinese)

36. Krasnosel'skii, MA: Positive Solutions of Operator Equations. Noordhoff, Groningen (1964)

37. Isac, G: Leray-Schauder Type Alternatives, Complementarity Problems and Variational Inequalities. Springer, Berlin (2006)

10.1186/1687-1847-2014-97

Cite this article as: Zhou et al.: Existence of multiple positive solutions for singular boundary value problems of nonlinear fractional differential equations. Advances in Difference Equations 2014, 2014:97 